\title{
Periodontal disease is a risk marker for coronary heart disease?
}

\author{
Abstracted from \\ Humphrey LL, Fu R, Buckley DI, Freeman M, Helfand M. \\ Periodontal disease and coronary heart disease incidence: a systematic review and meta-analysis. \\ J Gen Intern Med 2008; 23: 2079-2086 \\ Address for correspondence: Linda L Humphrey, Oregon Health and Science University, Mailcode BICC, \\ 3181 SW Sam Jackson Park Road, Portland OR 97239-3098, USA. E-mail: humphrey@ohsu.edu
}

\section{Question: Is periodontal disease an independent novel risk factor for incident coronary heart disease?}

Data Sources Relevant studies were identified using Medline and bibliographies of reviews, editorials, book chapters and letters discussing the relationship between periodontal disease and coronary heart disease (CHD).

Study selection Studies were assessed for inclusion by two reviewers. Prospective studies with cohort or nested case-control design with CHD or cardiovascular disease (CVD) as an outcome were included. Study quality was rated.

Data extraction and synthesis Data were abstracted by one reviewer and reviewed for accuracy by another author: any discrepancies were adjudicated by a third author. Meta-analysis was conducted to evaluate heterogeneity and publication bias.

Results Seven studies were included, some of which found that periodontal disease was independently associated with increased risk of CHD. Summary relative risk estimates for different categories of periodontal disease (including periodontitis, tooth loss, gingivitis and bone loss) ranged from 1.24 [95\% confidence interval $(\mathrm{Cl}), 1.01-1.51$ ] to $1.34(95 \% \mathrm{Cl}, 1.10-1.63)$. Risk estimates were similar in subgroup analyses by gender, outcome, study quality and method of periodontal disease assessment.

Conclusions Periodontal disease is a risk factor or marker for CHD that is independent of traditional CHD risk factors, including socioeconomic status. Further research is warranted in this important area of public health.

\section{Commentary}

The difficulty in assessing periodontal disease as a risk factor for to CHD has always been the result of standardised indices. Where one study relies almost exclusively on bone loss, another may only have the resources to examine tooth loss. Comparing one study with another directly may seem difficult, but if you look at the results of many similar studies, regardless of indices, each indicating a similar trend regardless of the disease marker chosen, the scientific community cannot help but give this tide of evidence some credence. Thus is the situation with proving an association between periodontal disease and CHD.

The evidence suggests that periodontal disease may be related to CHD by an oft theorised but as yet unproven pathway. Furthermore, research has reflected a belief that this association may be bidirec- tional, indicating that both disease processes may result from a common inflammatory pathway. This would seem to suggest that CHD, a disease with huge public health implications in morbidity and mortality, could be alleviated if the relationship could be more thoroughly extrapolated. Doing so could yield a treatment that was beneficial to not one but several ailments simultaneously.

This meta-analysis conducted by Humphrey and colleagues aims to explore the various investigations into the possible bidirectional causality between periodontal disease and CHD, and manages to do so despite the aforementioned lack of a standard index for either, having detailed those limitations accurately and fairly. Despite the discrepancy, the analysis has identified that a good deal of evidence exists that consistently reveals a statistically significant association between periodontal disease and CHD. A commendable feature that separates this study from others is the weighting they gave to the quality of evidence, rather than accepting results at face value.

Another aspect contained here is an attempt to explain the association from a biological standpoint, considering the possibility that a genetic inflammatory predisposition could account for the association, without ruling out the part that could be played by confounding factors such as smoking, diabetes and socioeconomic status, as well. The evidence has been approached from an unbiased and prospective view, and various alternative theories have been explained and argued fairly without being dismissed prematurely. Nonetheless, the analysis supports the view that periodontal disease is implicated in CHD with some degree of confidence. Such evaluation should continue in assessing the true bidirectional nature of these conditions, as most of the literature assessed seemed to focus on the causation of CHD without considering the possible dynamic between it and periodontal disease.

Aaron Cronin

Maxillofacial Unit, Sherwood Forest Hospitals NHS Trust, King's Mill Hospital, Sutton in Ashfield, Nottinghamshire, UK

Evidence-Based Dentistry (2009) 10, 22. doi:10.1038/sj.ebd.6400634 